\title{
CORE LEVEL SPECTROSCOPY AT SURFACES AND INTERFACES
}

\author{
F.J. HIMPSEL
}

IBM Research Division, Thomas J. Watson Research Center P.O. Box 218, Yorktown Heights, NY 10598, USA

\begin{abstract}
This article provides the basics of core level photoelectron spectroscopy, together with applications in the area of semiconductors. The use of synchrotron radiation is emphasized, particularly the opportunities that are opening up at the new, third generation light sources.
\end{abstract}

PACS numbers: $73.20 .-\mathrm{r}$

\section{Introduction}

Core levels are involved in a variety of spectroscopies. For example, optical absorption from a core level probes transitions into unoccupied valence states localized at a particular atom. Likewise, optical emission due to transition from occupied valence states into a core hole probes the local density of occupied states. Here we will mainly consider the spectroscopy of photoelectrons emitted from a core level by soft X-rays. The binding energy of the core level is directly reflected in the kinetic energy of the photoelectrons, as shown in Fig. 1 for a molecule containing several inequivalent carbon atoms [1]. This ability of revealing the chemical state of an atom has made this type of X-ray photoelectron spectroscopy (XPS), or electron spectroscopy for chemical analysis (ESC) extremely popular and has gained Kai Siegbahn, one of its main proponents, the Nobel prize in Physics. Traditionally, X-ray tubes emitting $\mathrm{Al} K_{\alpha}(1.49 \mathrm{keV})$ or $\mathrm{Mg} K_{\alpha}(1.25 \mathrm{keV})$ radiation have been utilized to excite the core levels. This leads to relatively energetic photoelectrons, which typically penetrate $20-30 \AA$ through a solid. The use of synchrotron radiation has opened up several new avenues. Apart from providing better resolution and intensity, together with polarization, the most useful aspect of synchrotron radiation has been its tunability. By tuning the escape depth of the photoelectrons through its minimum at about $50 \mathrm{eV}$ kinetic energy (Fig. 2) [2] the technique has reached a surface sensitivity that makes the detection of a single surface layer a matter of routine. This surface sensitivity has been of great use to surface physics and surface chemistry and has made this technique one of the most popular experiments performed at soft X-ray synchrotron light sources. For 


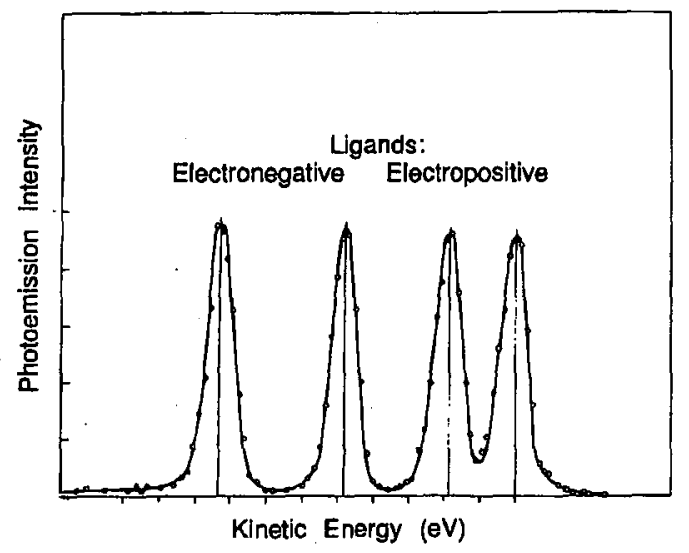

Fig. 1. Schematic of the charge-induced core level shift at a carbon atom induced by ligands of different electronegativity. Fluorine with high electronegativity removes electrons and creates a positive electrostatic potential that increases the binding energy of the C $1 s$ level (compare Ref. [1] for data).

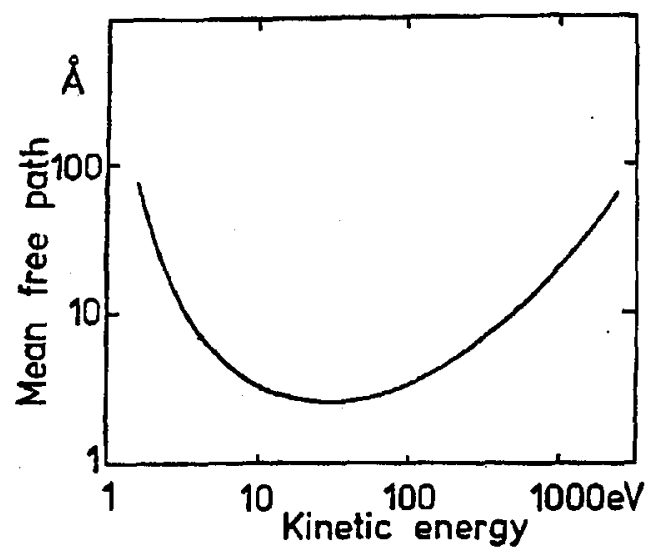

Fig. 2. Universal curve for the escape depth of photoelectrons versus kinetic energy. At high energy electrons become more penetrating, as expected intuitively from their speed. Counterintuitively, the escape depth increases again at low energies, where high energy excitation, such as plasmons and excitons become energetically inaccessible. The minimum escape depth at $30-50 \mathrm{eV}$ kinetic energy is in the order of a monolayer. For data on semiconductors see Ref. [2].

example, all the systematic work performed over the last decades by XPS on hydrocarbon molecules can now be applied to study their surface chemistry. In order to figure out the required photo energy range and energy resolution it is useful to look at the sharpest core levels of all the elements (Fig. 3) and their widths. These are the $1 s, 2 p, 3 d$, and $4 f$ shells, as they become core levels. Their binding energies 


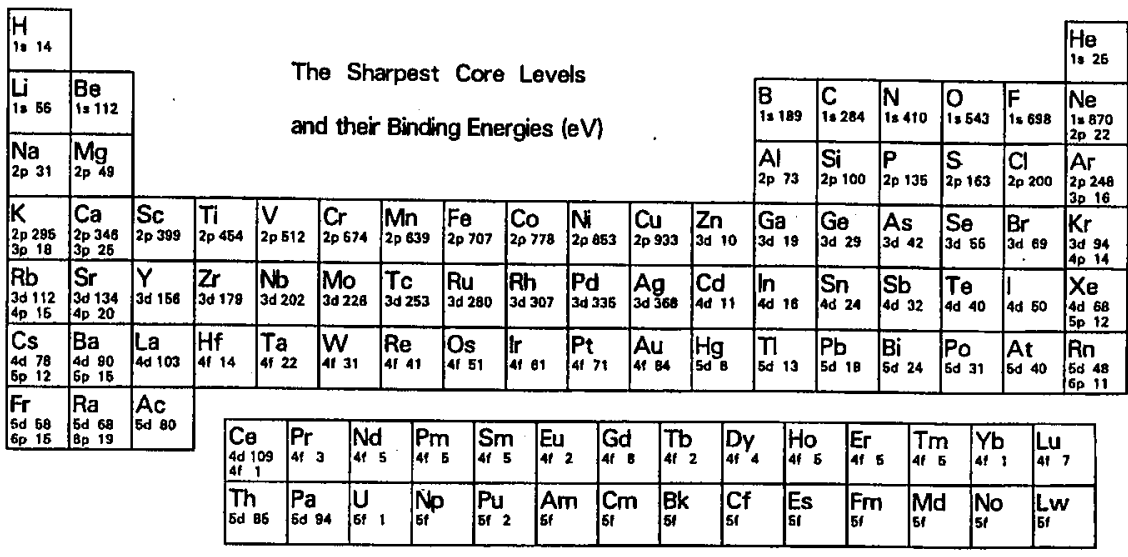

Fig. 3. Periodic Table containing the sharpest core level of each element and its binding energy (in eV). To excite these core levels photon energies of $100-1000 \mathrm{eV}$ are sufficient, which explains in large part the designed spectral range of soft X-ray synchrotron light sources.

are all less than $1000 \mathrm{eV}$, their intrinsic Auger lifetime widths are about $1 / 3000$ of the binding energy or more. These requirements have had a significant impact on the design parameters of the new, third generation synchrotron light sources. In particular, the photon energy range from $200-1000 \mathrm{eV}$ is not accessible with the required photon and electron resolution by previous light sources. It contains important sections of the Periodic Table, e.g. C, N, O, F, i.e. the elements of organic chemistry, and most of the metals and magnetic materials.

\section{Energy shifts}

\subsection{Initial versus final state effects}

Core level shifts can have a variety of causes, but inclusion of all of them in a comprehensive theoretical treatment has been achieved only in a few cases. However, core level spectroscopy has been so useful because the predominant contribution usually comes from a single effect, i.e. the charge transfer, or chemical effect. It is illustrated in Fig. 4. A charge transferred into or out of a valence orbital by chemical bonding introduces a change in the electrostatic potential at the core, which is inversely proportional to the average radius of this orbital according to Coulomb's law. Of course the opposite charges introduced at neighbor atoms in this process also cause electrostatic potentials at the core, but they are weaker due to the larger distances. This correcting potential has been considered in some cases [3], where crystalline order allows the summation of all contributior s to so-called Madelung energy.

The simplicity of this charge transfer concept is shown in Fig. 5, where each $\mathrm{F}$ ligand is found to introduce the same core level shift. This holds for free molecules as well as for surfaces. The shift of $2.8 \mathrm{eV}$ per $\mathrm{C}-\mathrm{F}$ bond is more than twice as large 


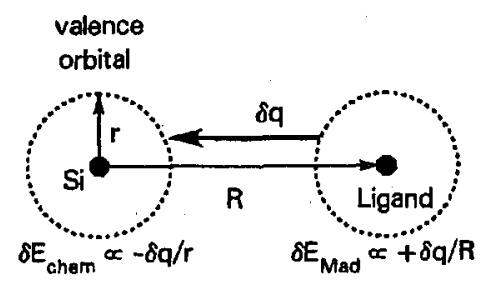

Fig. 4. Initial state effect on the core level shift. An electronic charge $\delta q$ transferred from a ligand to a valence orbital induces an electrostatic potential $-\delta q / r$ at the core, where $r$ is the radius of the valence orbital. The complementary charge $-\delta q$ at the ligand induces an opposite, but smaller potential $+\delta q / R$ at the core, where $R$ is the distance to the ligand. It corresponds to the Madelung energy.
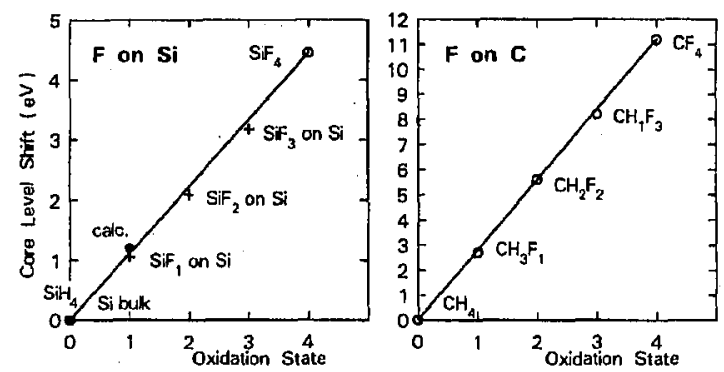

Fig. 5. Linear relation between the core level shift and number of fluorine neighbors for $\mathrm{Si}$ and $\mathrm{C}$. Due to the smaller size of the carbon atom (see Fig. 4) the shift per F is much larger for $\mathrm{C}$ than for $\mathrm{Si}(2.7 \mathrm{eV}$ versus $1.1 \mathrm{eV})$. From Ref. [4].

as that of $1.1 \mathrm{eV}$ per $\mathrm{Si}-\mathrm{F}$ bond. This is due to the small size of the $\mathrm{C}$ atom (see Fig. 4). Therefore, the study of chemical shifts becomes particularly attractive for organic compounds and their chemical reactions at surfaces. In semiconductors one observes a peculiar core level shift, which is due to the variable position of the Fermi level in the band gap (the Fermi level is the reference for the spectrometer, but the core level is at a fixed distance from the valence band maximum). Therefore, any change in the position of the Fermi level relative to the valence band shows up as an apparent core level shift. This effect can easily be distinguished from the chemical shift of surface atoms by tuning the probing depth with variable photon energy (see Ref. [5]). It has been used to determine the Schottky barrier and the band offset at semiconductor-metal contacts and heterojunctions, respectively.

All the core level shifts discussed so far can be considered as initial state effects, i.e. they exist already before the core hole is created in the photoemission process. Additional, so-called final state effects are due to the response of the solid (or molecular) to the creation of a core hole. One may think of a screening charge rushing in towards the core hole. It occupies the lowest empty valence orbital 
(Fig. 6). The screening charge gains electrostatic energy in the core hole potential, dependent on the amount of charge transfer and the radius of the screening orbital. This energy gain is transferred to the photoelectron and causes it to escape with higher kinetic energy. In an energy diagram (Fig. 6) the screening orbital is unoccupied before the excitation, and is pulled partially below the Fermi level by the potential of the core hole. Thus, the availability of screening orbitals just above the Fermi level is crucial in determining how much charge becomes available for screening.

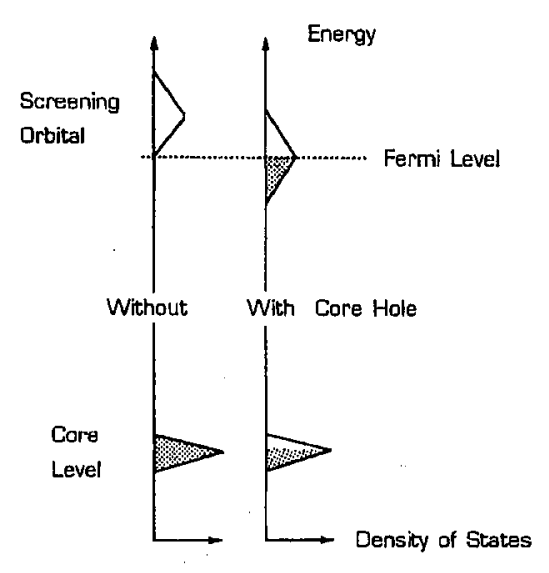

Fig. 6. Final state effect on the core shift. In response to the core hole created by the photoemission process a negative screening charge builds up in the lowest accessible screening orbital and shifts the electrostatic potential at the core up. The effectiveness of screening depends on the availability of a screening orbital close to the Fermi level.

The distinction of initial and final state effects is an intuitive concept, which can be clearly defined in any particular theory of the electronic structure and the photoemission process [6], although it may depend somewhat on the theoretical formalism (e.g. local density theory versus Hartree-Fock). Experimentally, it is non-trivial to distinguish the two contributions, since only a single shift is determined. There have been efforts to get additional information by using the energy shift of Auger electrons from the same core level as additional input. Another possibility opens up for the $4 f$ levels of rare earths, where the shift of the unoccupied part can be measured in addition to that of the occupied part, the former with inverse photoemission [9], the latter with photoemission (Fig. 7) [7, 8]. The initial state effects are expected to be independent of the occupation of the $4 f$ state, whereas the final state effects have opposite signs. The sign reversal can be rationalized by observing the effect of an extra electron, placed into an empty core level by inverse photoemission. It causes surrounding electrons to rush away, thereby emptying out a previously occupied screening orbital. Again, Coulomb energy is gained, which increases the energy of the emitted photon in inverse photoemission. The end result is that both occupied and empty core levels appear to be pulled towards the Fermi level by screening. 
Figure 7 shows first results on the shifts of occupied and empty Gd $4 f$ levels at a $\mathrm{Gd}(0001)$ surface, measured with photoemission and inverse photoemission, respectively. Surface-sensitive spectra at about $30 \mathrm{eV}$ kinetic energy exhibit shifted peak positions relative to bulk-sensitive spectra, obtained at $1.5 \mathrm{keV}$ electron energy (labelled XPS and BIS) [8]. The shifts are nearly equal for occupied and unoccupied $\mathrm{Gd} 4 f$, indicating that the initial state effect dominates. This is consistent with first principles calculations [10], which indicate that the initial effect dominates for metal surfaces, such as $\mathrm{Al}(100)$. Basically, surface and bulk are both completely screened in a metal due to the high density of screening electrons available near the Fermi level. This picture changes substantially at insulating surfaces. For example, oxygen induces opposite shifts of the filled and empty Gd 4f states [7]. Therefore, the simple chernical shift interpretation works best at surfaces of metals and semiconductors with high dielectric constant, but becomes risky at insulating surfaces.
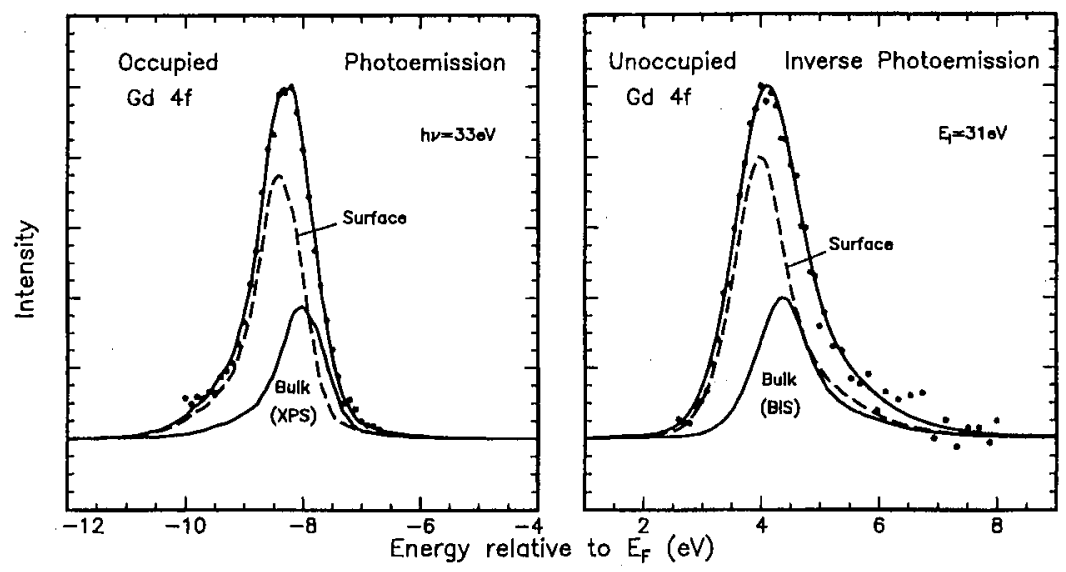

Fig. 7. Evaluation of initial versus final state effects in the surface core level shift of metallic $\mathrm{Gd}(0001)$. Photoemission spectra and inverse photoemission spectra provide the shifts for the filled and empty Gd $4 f$ level, respectively. Spectra at high electron energy (1.5 keV, labelled XPS and BIS) represent the bulk, spectra at low energy (about $30 \mathrm{eV}$ ) mostly the surface. Since both shifts are nearly equal, the initial state effect dominates. From Ref. [7].

\subsection{Applications to semiconductor surfaces and interfaces}

Figure 8 compares theoretical and experimental results for the core level shifts at the clean $\mathrm{Si}(100)$ surface $[11,12]$. These represent one of the few cases, where a state-of-the-art theoretical treatment of the core level shifts has been carried out, such that one is able to decompose the observed shifts into initial and final state components. The difficulty for calculations lies in the final state effect, 
where the core hole breaks the translation symmetry of the surface. The calculation predicts a buckled dimer structure for the $\mathrm{Si}(100)$ (Fig. 8), which consists of a negatively-charged up atom and a positively-charged down atom. Consequently, the calculated initial state shifts for these two atoms are $u_{1}$ wards and downwards in energy respectively (top panel in Fig. 8). Adding the final state effect causes an upwards shift for both core levels (middle panel) and produces good agreement with experiment (bottorn panel). The upwards shift due to the final state is surprising at first, since one might expect less screening charge to be available at the surface. Here, the energy position of the screening orbitals comes into play. It lies closer to the Fermi level at the surface, since there are empty surface states in the gap. They are more easily pulled below the Fermi level in the presence of the core hole than the bulk conduction band minimum, thus become more effective in accepting screening charge.

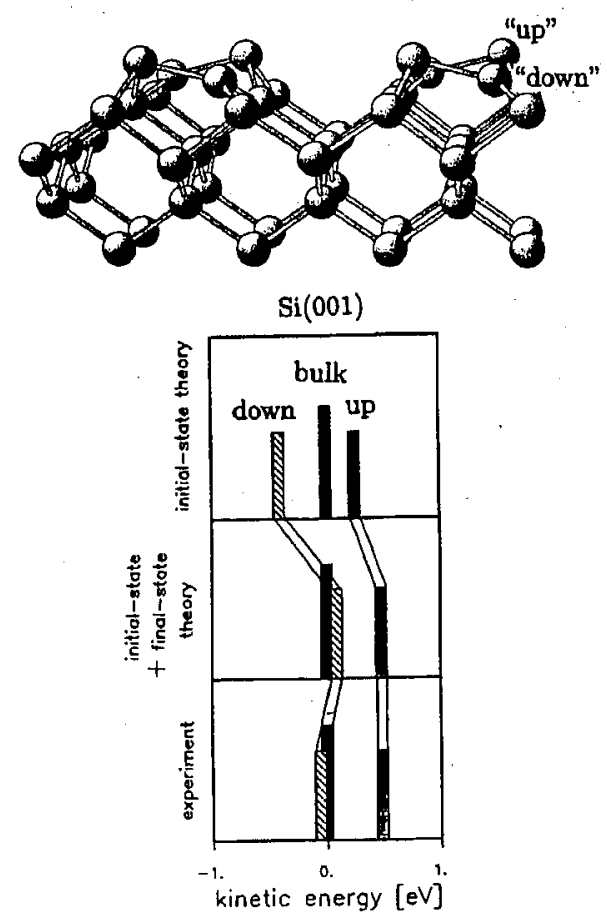

Fig. 8. First principles calculation of the initial and final state effect on the surface core level shifts of $\mathrm{Si}(100)$, and its comparison with the experiment. A model of the surface atom rearrangement into a buckled dimer structure is given. (Calculation: Ref. [11]; experiment: Ref. [12]).

In the area of interfaces we will discuss two examples, i.e. the epitaxial $\mathrm{CaF}_{2} / \mathrm{Si}(111)$ interface and the disordered $\mathrm{SiO}_{2} / \mathrm{Si}(100)$ interface. Fig are 9 [13] shows that the bond across the $\mathrm{CaF}_{2} / \mathrm{Si}(111)$ interface can be identified very easily with core level spectroscopy. After high temperature preparation $\left(700-800^{\circ} \mathrm{C}\right)$ 


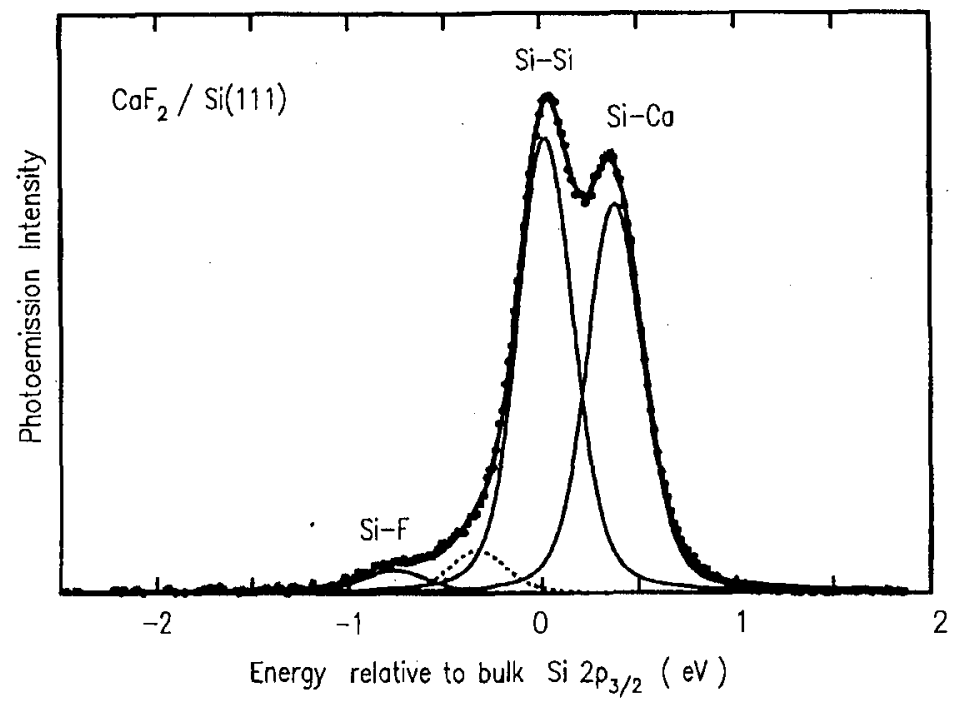

Fig. 9. Si $2 p_{3 / 2}$ core level spectrum of the $\mathrm{CaF}_{2} / \mathrm{Si}(111)$ interface, showing that $\mathrm{Si}$ bonds mainly to $\mathrm{Ca}$ at the interface. Compare Ref. [13].

the majority of the interface bonds connects $\mathrm{Si}$ with $\mathrm{Ca}$, producing an upwards shift of the Si $2 p$ level as for the pure Ca on Si. Only a small minority of Si-F bonds can be detected on the opposite side of the bulk line, probably due to defects.

The SiO. ji(100) interface exhibits extremely high electrical quality, despite its disordered nature that is caused by the arrorphous growth of $\mathrm{SiO}_{2}$. The density of electrically-active defects is less than $10^{-5}$ monolayers. Looking at the interface with depth-sensitive Si $2 p$ core level spectroscopy (Fig. 10) [14] one finds that all intermediate oxidation states of Si can be resolved. They are unstable in the bulk, where $\mathrm{SiO}$ disproportionates into $\mathrm{Si}$ and $\mathrm{SiO}_{2}$. Only the geometry of the interface, with $\mathrm{Si}$ on one side and oxide on the other, forces the Si interface atoms into these oxidation states. From the distribution of oxidation states at the interface one can try to make connections with electrical properties, despite the fact that the defect states are too much dilute to be seen directly. Indeed, different preparation conditions produce different distributions (Fig. 10 top versus bottom). Interfaces with higher $\mathrm{Si}^{1+}$ context and less $\mathrm{Si}^{3+}$ tend to give better electrical properties (Fig. 10 bottom). They are produced at high oxygen pressures and low temperatures, using very flat and clean $\mathrm{Si}(100)$ surfaces.

An emerging area of semiconductor surface chemistry is layer-by-layer, or "digital" growth and etching. Such methods promise to make the fabrication of semiconductor structures with atomic precision a matter of routine. Controlling semiconductor layers at the monolayer level has many interesting aspects. For example, there are dramatic changes in the band gap at heterointerfaces, e.g. by a factor of 2 or $1 / 5$ at the $\mathrm{CaF}_{2} / \mathrm{Si}(111)$ interface [15], depending on whether one compares with $\mathrm{Si}$ or $\mathrm{CaF}_{2}$. Superlattices with short periods consist almost exclusively of interfaces, making such structures a promising testing ground for 


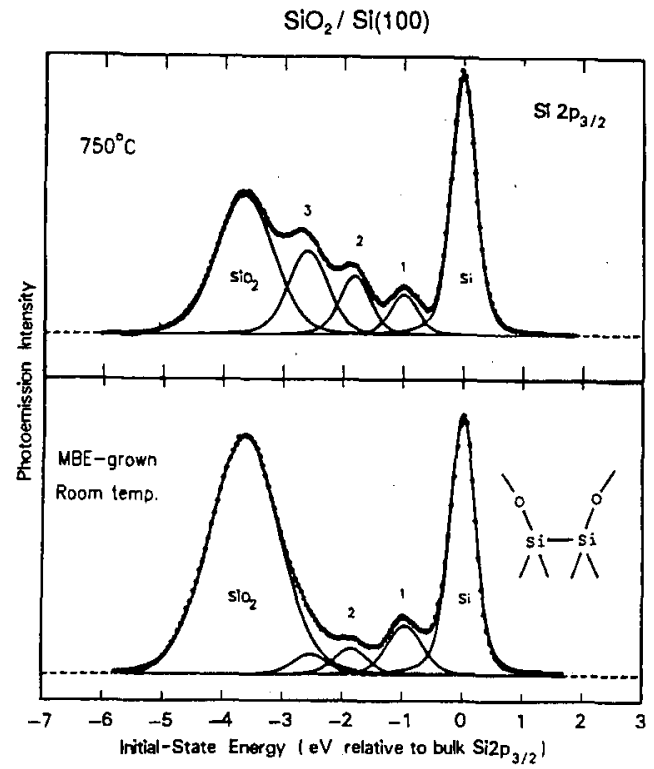

Fig. 10. Si $2 p_{3 / 2}$ core level spectra showing intermediate oxidation states of Si at $\mathrm{SiO}_{2} / \mathrm{Si}(100)$ interfaces. Their distribution changes with the preparation conditions, which in turn determine the electrical quality of the interface. At low temperatures the interface geometry is dominated by single $\mathrm{Si}-\mathrm{O}$ bonds, indicating a dimerization of $\mathrm{Si}$ interface atoms, as on the clean surface. After Ref. [14].

producing new materials with tailored electronic properties. Other ways of tailoring semiconductors are the introduction of dipole layers at heterojunctions to change the band offset [16], or the use of different stacking sequences, e.g. in $\mathrm{SiGe}$ and $\mathrm{SiC}$ to modify the band gap. An area where atomic-scale effects have macroscopic consequences is the magnetism of ferromagnet/noble metal multilayers. In this case the addition of a few layers to a noble metal spacer layer can switch the coupling between the ferromagnetic layers from parallel to antiparallel, and cause a "giant" magnetoresistance effect, which is very likely to be used for the readout of data from magnetic disks [17].

An example of the questions studied for layer-by-layer growth, or atomic layer epitaxy (ALE) is given in Fig. 11 [18]. Here, a process is being tested, where a monolayer of $\mathrm{Si}$ is deposited in self-limiting fashion by adsorption of $\mathrm{SiH}_{2} \mathrm{Cl}_{2}$. In a second step it is reactivated by reacting off the $\mathrm{Cl}$ passivation layer with $\mathrm{H}_{2}$ as $\mathrm{HCl}$. This cycle can be repeated over and over. While it seemed natural that $\mathrm{Cl}$ could passivate the Si surface after depositing a monolayer, and that $\mathrm{H}_{2}$ could reactivate it, the actual chemical reactions at the surface remained unknown. With Si $2 p$ core level spectroscopy (Fig. 11) it is easy to find out that the surface is indeed passivated by $\mathrm{Cl}$, although not by the $\mathrm{SiCl}_{2}$ groups expected from the two broken bonds per surface atorn on the truncated $\mathrm{Si}(100)$ surface. Instead, $\mathrm{SiCl}_{1}$ groups are seen, which indicate a dimerization of the Si surface atoms, as on clean $\mathrm{Si}(100)$. 


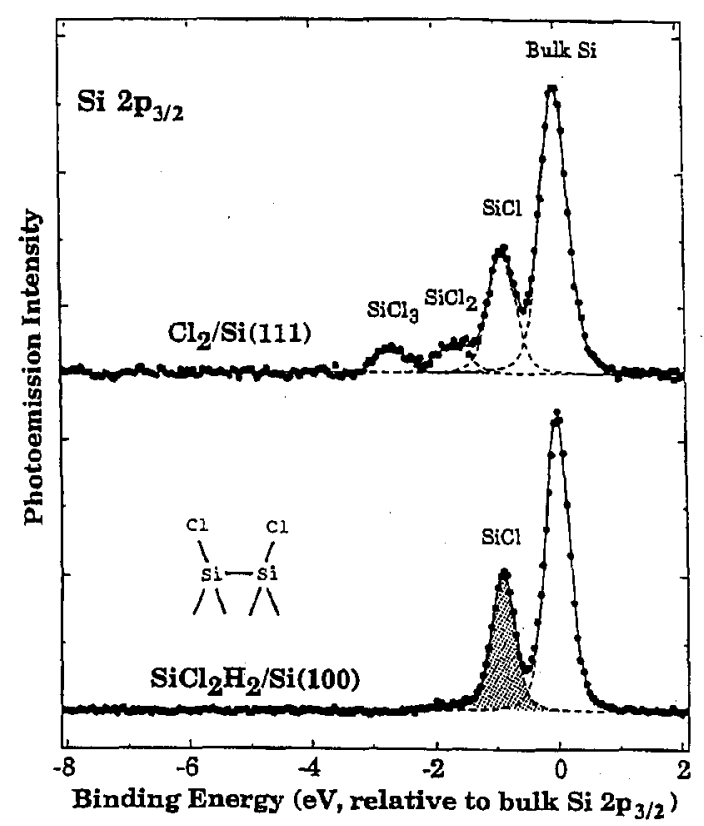

Fig. 11. Passivating of the $\mathrm{Si}(100)$ surface by $\mathrm{Cl}$ during atomic layer epitaxy with $\mathrm{SiH}_{2} \mathrm{Cl}_{2}$. Due to dimerization of the $\mathrm{Si}$ surface atoms only $\mathrm{SiCl}$, groups are observed, while a naive broken bond model would predict $\mathrm{SiCl}_{2}$ groups. From Ref. [18].

Thereby, half the broken bonds are tied up in the dimer bonds. The activation step with $\mathrm{H}_{2}$ turns out to be different from the expected $\mathrm{HCl}$ reaction. It occurs only with atomic hydrogen, or by thermal desorption of the $\mathrm{Cl}$ passivation layer.

\section{Intensities}

\subsection{Counting atomic layers}

Apart from the core level shift the experiment provides a second number, i.e. the intensity. In principle, it can be converted into an atom density, but there are many calibration factors to be taken into account [19]. By using a photoelectron spectrometer with exceptionally large angular acceptance (1.8 steradian [20]) one is able to average out most of the diffraction effects discussed in Sec. 3.2. Only EXAFS-like, energy-dependent intensity oscillations remain (usually less than 10\%). In addition, the atornic cross section of shifted core levels can differ substantially from the bulk in the energy region near the core absorption threshold $[5,14]$. This can be calibrated by comparing intensities above threshold (nearly $100 \mathrm{eV}$ ), where the cross sections are equalized. For example, the $\mathrm{Si}^{3+}$ has a 1.7 times higher cross section than bulk Si at a photon energy of $130 \mathrm{eV}$, i.e. near the escape depth minimum. After these corrections it is reasonable to predict layer thicknesses from the core level intensities within half an atomic layer. As an example for the contribution of the first Si layer to the total signal one can take 
the Ca-shifted Si $2 p$ line in Fig. 9, or the Cl-shifted Si $2 p$ in Fig. 11. They comprise $30-40 \%$ of the total signal, showing how surface-sensitive one can get using synchrotron radiation.

\subsection{Photoelectron diffraction and holography}

Measuring the angular distribution of core level photoemission it is possible to obtain structural information about the atoms surrounding a particular atom. The concept can be understood most elegantly by making the analogy to the creation of a hologram (Fig. 12) [21]. The directly-limited photoelectron wave can be viewed as a reference wave, whereas the waves backscattered from neighbor atoms correspond to the object wave. The total angular distribution of the photoemission intensity represents the hologram, which is the interference between the reference and object wave. In a computer, one can reconstruct the photoelectron hologram by "shining" the spherical reference wave onto the measured hologram, thereby obtaining constructive interference at the positions of neighbor atoms. In practice, there are two main hurdles to this concept. The reconstruction always produces an additional, conjugate image, since the phase information is not retained in the process. In addition, there are multiple scattering events at the electron energies in question $(100-1000 \mathrm{eV})$, which introduce artifacts into the reconstructed pic-

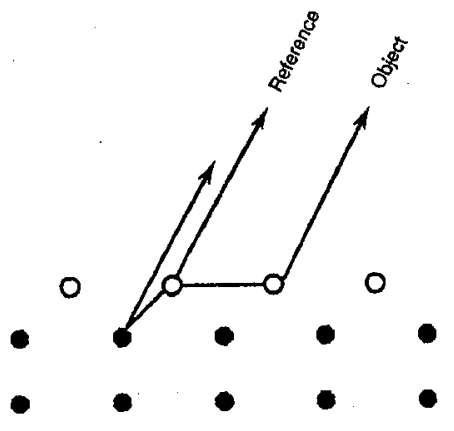

Fig. 12

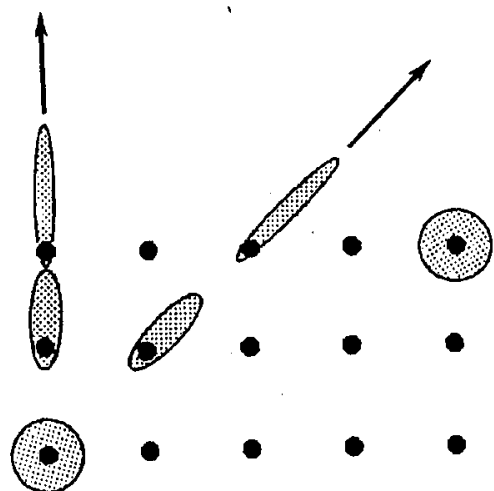

Fig. 13

Fig. 12. Schematic of photoelectron diffraction and photoelectron holography. The directly-emitted core level photoelectron represents the reference wave, the backscattered waves, the object, and the angular distribution of photoelectrons, the hologram. Numerical reconstruction reveals neighbor atom positions within the mean free path of the photoelectrons. Compare Ref. [21].

Fig. 13. Triangulation of the surface atom geometry by forward scattering of photoelectrons. Emission from bulk atoms is channeled along rows of atoms, whereas emission from surface atoms remains isotropic. 
ture. Both effects can be reduced significantly by averaging the reconstruction over holograms taken at different kinetic energies, making the use of synchrotron mandatory for this method [21]. Various averaging methods are still being tested, e.g. taking $k_{\|}$slices and then averaging over $k_{\perp}$ [21], or by taking first $k_{\perp}$ slices [22] and then sweeping $k_{\|}$. At present it appears that photoelectron holography will become a helpful technique for obtaining a first clue about the bond topology of unknown structures. For an exact determination of the bond lengths and angles it will be necessary to simulate the holograms with a full-fledged multiple scattering: calculation, as in LEED intensity analysis [23].

A much simpler aspect of photoelectron diffraction is the forward scattering observed at higher energies of typically $1000 \mathrm{eV}$ (Fig. 13). The higher the kinetic energy, the more the elastic scattering becomes focussed in the forward direction, leading to a "channelling" of the intensity along atomic rows. These relatively narrow intensity maxima have been used to determine the growth mode, registry and stacking of thin epitaxial films [24].

\section{Opportunities at 3rd generation light sources}

Third generation light sources are based on undulators, which boost the spectral brilliance of second generation (dedicated) source by a factor of $10^{4}$. Spectral brilliance, i.e. photons per solid angle and source area, represents the volume in phase space that the emitted photons occupy. According to Liouville's theorem, this volume cannot be decreased by the subsequent optics. Therefore, brilliance is the number to consider when synchrotron light has to be highly monochromatized or focussed. An efficient monochromatization is important for doubly-dispersing experiments, such as photoelectron spectroscopy and fluorescence spectroscopy. At second generation sources spectroscopy it is not practical to perform core level spectroscopy at a resolution smaller than the Auger lifetime width if the core level is deeper than $200 \mathrm{eV}$. Looking at the Periodic Table in Fig. 3 one finds that the "organic" elements $\mathrm{C}, \mathrm{N}, \mathrm{O}, \mathrm{F}$, are in this category, as well as the $3 d$ and $4 d$ transition and noble metals, including the common magnetic materials $\mathrm{Cr}, \mathrm{Mn}$, $\mathrm{Fe}, \mathrm{Co}, \mathrm{Ni}$. Therefore, one can expect a widespread popularity of organic surface chemistry, polymers, and magnetic materials at third generation sources.

Core level fluorescence needs photons even more than photoemission, since the fluorescence yield is typically down by three orders of magnitude from that for photoelectron and Auger emission for the sharp core levels listed in Fig. 3. Particularly the observation of resonance effects in fluorescence and the detection of submonolayer quantities is just becoming feasible with undulators. Despite its low cross section, fluorescence detection offers unique advantages. The secondary electron background that is always present in electron spectroscopies does not interfere with fluorescence, making it possible to detect very dilute quantities of an element, given enough photons. Core level fluorescence is already routinely used for this purpose in the hard X-ray regime, where the cross-sections are higher and the detection easier. The deep penetration of soft $\mathrm{X}$-ray photons allows it to look at buried interfaces and at surface chemical reactions through the ambient reactant. Some first results from a third generation undulator source are shown in Fig. 14 [25]. Here, the fluorescence spectrum of a buried boron monolayer is 
detected. When tuning the excitation energy to the lowest B $1 s$-to- $\pi^{*}$ resonance, an intense additional peak is observed, which is caused by direct recombination from the $\pi^{*}$. Such phenomena make it possible to detect small amounts of boron at surfaces and buried interfaces. A wide range of applications in the analysis of silicon devices is waiting to be explored, since B is the common $p$-type dopant in Si.

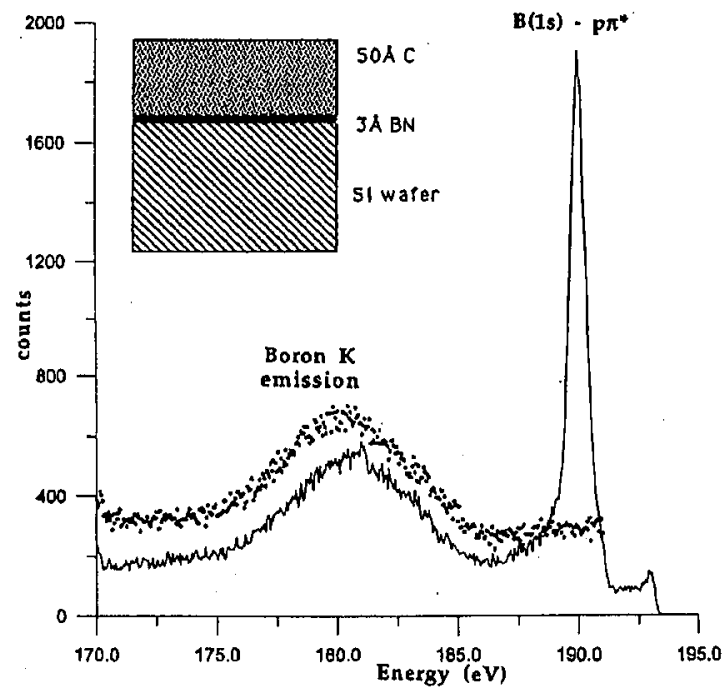

Fig. 14. Fluorescence spectrum of a buried monolayer of $\mathrm{BN}$ at excitation energies above and below the lowest $\mathrm{B} 1 s$-to-pi* resonance. The resonant enhancement promises sensitive detection of B dopants in the Si devices. From Ref. [25].

Perhaps the best match between the capabilities of third generation light sources and the specific experimental demands can be found for element-resolving microscopy techniques. A variety of schemes have been tried, which use either photons or photoelectrons for imaging and may be designed for scanning or imaging data acquisition. Elemental contrast can be achieved in two ways. The first involves a kinetic energy analysis of the photoelectrons, thereby picking out different core level peaks for imaging. The second uses tunable photon energy to acquire pictures above and below a core level absorption edge. Existing microscopes [26] have already produced a wealth of information about nucleation and growth, defect formation, and inhomogeneous chernical reactions at surfaces. With current low energy electron diffraction microscopes it has become routine to achieve resolutions down to the $100 \AA$ level. Similarly-designed imaging photoelectron microscopes have to deal with chromatic abberations. These can be overcome either by an energy filter (which is difficult to build at the required resolution), or by compensating reflecting electron optics (which is still under development). If these technical questions can be solved, photoemission microscopes promise to have even wider applications than electron diffraction microscopes, since they are not limited to ordered substrates and provide additional chemical information. 


\section{References}

[1] U. Gelius, E. Basilier, S. Svensson, T. Bergmark, K. Siegbahn, J. Electr. S'pectrosc. Rel. Phen. 2, 405 (1974).

[2] H. Gant, W. Moench, Surf. Sci. 105, 217 (1981).

[3] G. Hollinger, P. Kumurdjian, J.M. Mackowski, P. Pertosa, L. Porte, Tran Minh Duc, J. Electron Spectrosc. 5, 237 (1974); J.Q. Broughton, P.S. Bagus, J. Electron Spectrosc. 20, 261 (1980); J.W. Davemport, R.E. Watson, M.L. Perlman, T.K. Sham, Solid State Commun. 40, 999 (1981) and Phys. Rev. B 24, 1791 (1981).

[4.] F.J. Himpsel, Appl. Phys. A 38, 205 (1985). For F on diamond a C $1 s$ shift of $1.85 \mathrm{eV}$ (relative to the bulk) and $2.6 \mathrm{eV}$ (relative to the clean surface) has been found, see J.F. Morar, F.J. Himpsel, G. Hollinger, J.L. Jordan, G. Hughes, F.R. McFeely, Phys. Rev. B 33, 1340 (1986).

[5] F.J. Himpsel, B.S. Meyerson, F.R. McFeely, J. Morar; A. Taleb-Jbrahimi, J.A. Yarmoff, in: Proc. of the Enrico Fermi School on Photoemission and Absorption Spectroscopy of Solids and Interfaces with Synchrotron Radiation, Eds. M. Campagna, R. Rosei, North-Holland, Amsterdam 1990, p. 203.

[6] R.E. Watson, M.L. Perlman, J.F. Herbst, Phys. Rev. B 13, 2358 (1976); A.R. Williams, N.D. Lang, Phys. Rev. Lett. 40, 954 (1978); M. Methfessel, D. Hennig, M. Scheffler, Surf. Sci. 287-288, 785 (1993).

[7] J.E. Ortega, F.J. Himpsel, Dongqi Li, P.A. Dowben, Solid State Commun., in press; Compare also A.V. Fedorov, E. Arenholz, K. Starke, E. Navas, L. Baumgarten, C. Laubschat, G. Kaindl, to be published.

[8] J.K. Lang, Y. Baer, P.A. Cox, J. Phys. F 11, 21 (1981).

[9] F.J. Himpsel, Surf. Sci. Rep. 12, 1 (1990).

[10] P.J. Feibelmall, Phys. Rev. B 39, 4866 (1989).

[11] E. Pellke, M. Scheffler, Phys. Rev. Lett. 71, 2338 (1993).

[12] E. Landemark, C.J. Karlsson, Y.-C. Chao, R.I.G. Uhrberg, Phys. Rev. Lett. 69, 1588 (1992).

[13] D. Rieger, F.J. Himpsel, U.O. Karlsson, F.R. McFeely, J.F. Morar, J.A. Yarmoff, Phys. Rev. B 34, 7295 (1986).

[14] F.J. Himpsel, F.R. McFeely, A. Taleb-Ibrahimi, J.A. Yarmoff, G. Hollinger, Phys. Rev. B 38, 6084 (1982); F.J. Himpsel, D.A. Lapiano-Smith, J.F. Morar, J. Berk, in: The Physics and Chemictry of $\mathrm{SiO}_{2}$ and the SiO 2 Interface 2, Eds. C.R. Helms, B.E. Deal, Plenum Press, New York 1993, p. 237.

[15] T.F. Heinz; F.J. Himpsel, E. Palange, E. Burstein, Phys. Rev. Lett. 63, 644 (1989).

[16] G. Bratina, L. Sorba, A. Antonini, G. Biasiol, A. Franciosi, Phys. Rev. B 45, 4528 (1992).

[17] For an overview on magnetic reading heads see: R.E. Jones, C.D. Mee, in: Magnetic Recording: Technology, Eds. D. Mee, E.D. Daniel, Vol. 1, Mc Graw Hill, New York 1987 , p. 244. For giant magnetoresistance, spin valves, and oscillatory magnetic coupling see: S.S.P. Parkin, Phys. Rev. Lett. 67, 3598 (1991); B. Dieny, V.S. Speriosu, S.S.P. Parkin, B.A. Gurney, D.R. Wilhoit, D. Mauri, Phys. Rev. B 43, 1297 (1991). For quantized electronic states in these structures see: J.E. Ortega, F.J. Himpsel, G.J. Mankey, R.F. Willis, Phys. Rev. B 47, 1540 (1993) and J. Appl. Phys. 73, 5771 (1993). 
[18] J.A. Yarmoff, D.K. Shuh, T.D. Durbin, C.W. Lo, D.A. Lapiano-Smith, F.R. McFeely, F.J. Himpsel, J. Vac. Sci. Tecnol. A 10, 2303 (1992).

[19] A. Jabłoński, Acta Phys. Pol. A 86, 787 (1994).

[20] D.E. Eastman, J.J. Donelon, N.C. Hien, F.J. Himpsel, Nucl. Instrum. Methods 172, 327 (1980).

[21] J.J. Barton, Phys. Rev. Lett. 61, 1356 (1988); L.J. Termnello, J.J. Barton, D.A. Lapiano-Smith, Phys. Rev. Lett. 70, 599 (1993).

[22] H. Wu, G.J. Lapeyre, H. Huang, S.Y. Tong, Phys. Rev. Lett. 71, 251 (1993).

[23] A.M. Bradshaw, J. Electron Spectrosc. Relat. Phenom. 62, 33 (1993).

[24] C.S. Fadley, in: Synchrotron Radiation Research: Advances in Surface Science, Ed. R.Z. Bachrach, Plenum Press, New York 1992, Ch. 11; B. Eisenhut, J. Stober, G. Rangelov, Th: Fauster, Phys. Rev. B 47, 12980 (1993).

[25] J.A. Carlisle, L.J. Terminello, E.A. Hudson, R.C.C. Perera, J.H. Underwood, T.A. Callcott, J.J. Jia, F.J. Himpsel, submitted to Appl. Phys. Lett.

[26] M. Mundschan, E. Bauer, W. Swiech, J. Appl Phys. 65, 581 (1989); H.H. Rotermund, S. Jakubith, A. von Oertzen, G. Ertl, Phys. Rev. Lett 66, 3083 (1991); J. Stohr, Y. Wu, B.D. Hermsmeier, M.G. Samant, G.R. Harp, S. Koranda, D. Dunlam, B.P. Tonner, Science 259, 658 (1993). 